\title{
Sensing within the OTDR dead-zone using a two-mode fiber
}

\author{
Yuan Mao ${ }^{1, \# \text {, Islam Ashri }}{ }^{1, \#}$, Biwei Wang ${ }^{1,2}$, Frode Hveding ${ }^{3}$, Ahmed Y. Bukhamseen ${ }^{3}$, \\ TienKHEe NG ${ }^{1}$, AND BOON S. OOI ${ }^{1, *}$ \\ ${ }^{1}$ King Abdullah University of Science and Technology (KAUST), Computer, Electrical, and Mathematical Sciences and Engineering, Thuwal 23955-6900, Saudi \\ Arabia \\ ${ }^{2}$ Department of Electronic and Information Engineering, The Hong Kong Polytechnic University, Hong Kong SAR, China \\ ${ }^{3}$ EXPEC Advanced Research Center, Saudi Aramco, Dhahran 34464, Saudi Arabia \\ \# These authors contributed equally to this work \\ *Corresponding author: Boon.ooi@kaust.edu.sa
}

Compiled May 8, 2020

Optical time-domain reflectometer (OTDR) is incapable of providing sensing or diagnostic information within dead-zones. We use a two-mode fiber (TMF) and a photonic lantern to completely overcome the main OTDR's dead-zone originated from the front facet of optical fiber. This is achieved via injecting the optical pulses of the OTDR in the form of the fundamental $\mathrm{LP}_{01}$ mode, and meanwhile collecting the Rayleigh signals associated with the higher-order modes. Using the reported TMF-based OTDR, we accurately sense the position and frequency of a vibration event located within the dead-zone, as a proof-of concept demonstration. () 2020 Optical Society of America

http://dx.doi.org/10.1364/ao.XX.XXXXXX

Optical time-domain reflectometer (OTDR) is a popular instrument used to characterize optical fibers [1]. Particularly, OTDR can measure losses and locate faults along a fiber under test (FUT). Additionally, OTDR has been deployed in a myriad of distributed fiber optic sensing systems [2, 3]. For instance, OTDR-based distributed sensors can measure vibration [4], temperature [5], strain [6], etc., along an optical fiber. Consequently, OTDR is important for many industrial applications, including oil and gas industry [7] and structural health monitoring [8].

The available commercial OTDR systems suffer from the existence of dead-zones along a FUT due to intense Fresnel reflections generated by fiber front facet, connectors, and other optical components. The large difference of power between these Fresnel reflections and the backscattered Rayleigh light results in saturating the receiver and creating dead-zones. Depending on the input optical pulses' width and photodetector's bandwidth, dead-zone is typically tens to hundreds of meters [4]. Within a dead-zone region, conventional OTDR is unable to provide any reliable sensing or diagnostic data. For OTDR-based distributed fiber optic sensing applications, such as distributed acoustic sensing (DAS), a single and continuous fiber optic cable is typically deployed $[9,10]$. Under this scenario, the distributed sensor includes an inevitable main dead-zone resulted from the fiber front facet's Fresnel reflection. Missing sensing information, in even a short section along the optical fiber, is considered a severe problem for critical applications, such as perimeter intrusion detection and security using DAS [11].

To encounter the effect of the front facet's dead-zone, a dummy fiber, named launch cable, can be added between OTDR unit and FUT [12]. In this case, the dead-zone occurs within the dummy optical fiber instead of the FUT, so that near-end events are more readily detectable. This technique, however, reduces the maximum sensing range of OTDR system. Additionally, the dummy fiber should have a protective jacket and correct connectors on each end which may become very expensive since one launch cable would be needed for every possible connectors combination. The connector between the dummy fiber and FUT might create also another dead-zone within the beginning of the FUT. Alternatively, the time-correlated single photon-counting (TC-SPC) OTDR has been reported to overcome the limitations of dead-zones by detecting the spontaneous Stokes Raman signal [13]. Unfortunately, the TC-SPC OTDR cannot be used for sensing applications that rely on Rayleigh scattering. Digital signal processing (DSP) has also been introduced to discard the impact of dead-zones [14]; however, DSP-enhanced OTDRs are relatively complicated systems.

We here use a two-mode fiber (TMF) and a photonic lantern mode-division-multiplexer/demultiplexer (MUX/DEMUX) to design an OTDR system free of the dead-zone originated from the fiber front facet. Although single-mode fiber (SMF) is the preferred platform for sensing applications, the operation characteristics of SMF-based sensors cannot be easily tuned. Additionally, it is difficult to deploy a SMF-based sensor that discriminates multiple sensing parameters. For instance, conventional SMF-based Brillouin optical time-domain reflectometer (BOTDR) resolves either strain or temperature along the fiber [2]. Consequently, few-mode fiber (FMF) has recently attracted significant research interest owing to its unique capabilities which can be utilized in many applications including multi-parameter [15], OTDR-based [16], and absorption-based sensing [17]. The reported work here would be significantly beneficial for the emerging FMF-based optical sensors. 
A comparison between the typical SMF-based OTDR system [18] and our reported FMF-based one is shown schematically in Fig. 1. In the SMF-based OTDR [Fig. 1(a)], optical pulses are injected through a SMF-based circulator into a SMF under test. As the fundamental mode $\left(\mathrm{LP}_{01}\right)$ propagates in the pump direction along the SMF under test, two different kinds of light signals are reflected/scattered in the backward direction. The first signal type is that produced by Fresnel reflections, occur inside the circulator and at the connector. The second type of the backward light is the outcome of the elastic Rayleigh scattering phenomenon occurred along the optical fiber [18]. We do not include the inelastic Brillouin and Raman scattering in this study because they are not impacted by dead-zones. Both of the Fresnel reflections and backscattered Rayleigh signal are in the form of the $\mathrm{LP}_{01}$ mode which results in creating a deadzone at the receiver, as shown in Fig. 1(a). In a multimode fiber (MMF-) based OTDR, the dead-zone's effect still exists because of launching many spatial modes in the pump direction, and detecting the entire modes in the backward direction.

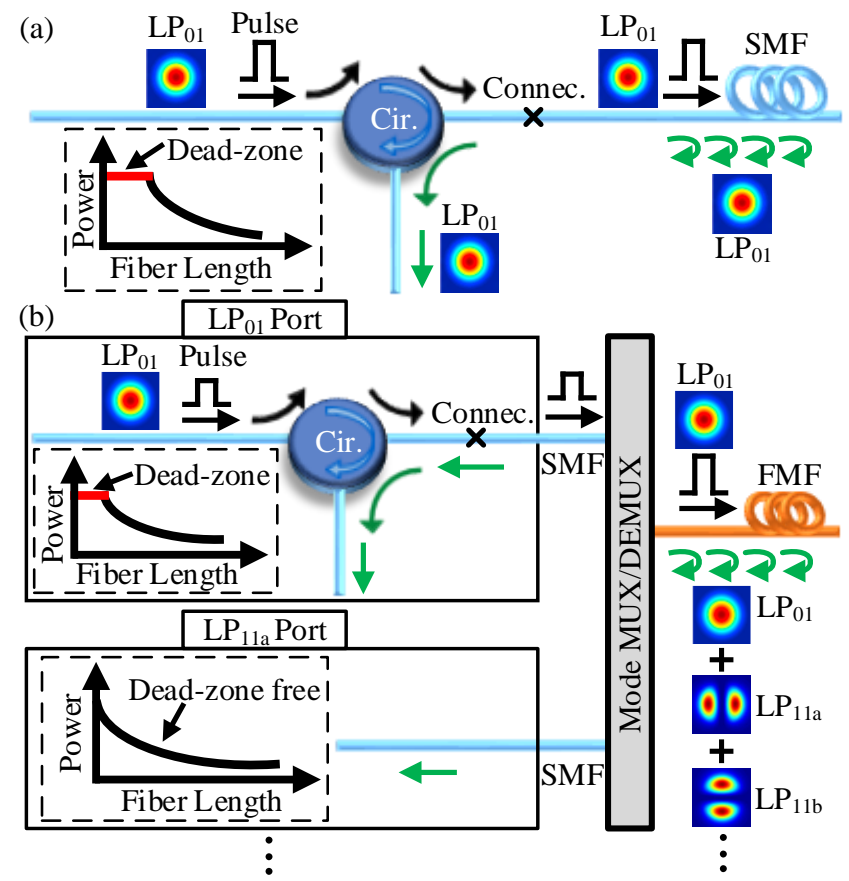

Fig. 1. Comparison between the typical OTDR (a) and the reported FMF-based OTDR (b). Cir., circulator; Connec., connector.

In contrast, our reported FMF-based OTDR is shown in Fig. 1(b) where a photonic lantern mode MUX/DEMUX is added between the transmitter side and a FMF under test. Assuming the used FMF supports propagating $N$ modes, the mode MUX/DEMUX should have $N$ input SMF ports and a single output FMF port [19]. Considering the representative example shown in Fig. 1(b), we launch the optical pulses from the input port of the $\mathrm{LP}_{01}$ mode such that the mode MUX delivers the $\mathrm{LP}_{01}$ pulses to the FMF. In the backward direction, Fresnel reflections originated within the circulator and at the connector occur only for the injected $\mathrm{LP}_{01}$ mode while the Rayleigh scattering excites all of the modes supported by the FMF [20]. Since the mode DEMUX decomposes the backscattered Rayleigh signal into individual modes, the recorded signals associated with the higher-order modes $\left(\mathrm{LP}_{11 \mathrm{a}}, \mathrm{LP}_{11 \mathrm{~b}}\right.$, etc.) are dead-zone free. These higher order modes can offer reliable OTDR functionalities along the entire FMF, even within the dead-zone region of the $\mathrm{LP}_{01}$ mode. This design can be generalized such that any mode can be used for the pulse injection, and the other modes become dead-zone free.

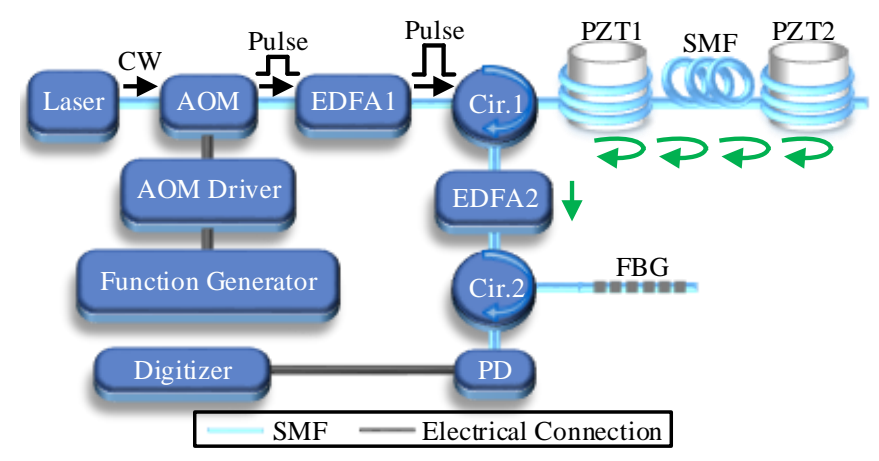

Fig. 2. Experimental setup of the typical OTDR-based DAS designed using a SMF.

To validate our approach, we compare the performances of two DAS systems designed using the phase-sensitive SMF- and TMF-based OTDR. Fiber optic DAS is primarily used to detect vibrations along a FUT using Rayleigh scattering [4]. The experimental setup of the typical SMF-based DAS is shown schematically in Fig. 2 where a narrow linewidth $(100 \mathrm{~Hz})$ laser generates a continuous wave (CW) light of a $40-\mathrm{mW}$ optical power and a 1535-nm wavelength. Using an acousto-optic modulator (AOM) driven by a function generator, the $\mathrm{CW}$ light is converted into optical pulses with a $30-\mathrm{ns}$ width and a $20-\mathrm{kHz}$ repetition rate. The optical pulses are then amplified by an erbium-doped-fiberamplifier (EDFA1) to have a $\sim 30-\mathrm{dBm}$ peak power and next the pulses are injected throughout a circulator (Cir.1) into a standard SMF of a $\sim 2.1-\mathrm{km}$ length. Starting at a $\sim 2-\mathrm{m}$ separation from the input port of the SMF, we wind a 2-m section of the SMF around a vibrating piezoelectric transducer (PZT1) cylinder. The PZT1's vibration is located within the front dead-zone of the OTDR-based DAS. Near the fiber end, a 2-m section of the SMF is wound around another PZT cylinder (PZT2). Both of PZT1 and PZT2 are similar and driven with the same function generator such that they vibrate with identical amplitude and frequency ( $5 \mathrm{kHz}$ here). As the pump optical pulses propagate along the SMF, the backscattered Rayleigh signals are guided via the Cir.1 towards another erbium-doped-fiber-amplifier (EDFA2). The amplified spontaneous emission (ASE) noise of the EDFA2 is filtered out using a fiber Bragg grating (FBG), and the filtered signal is respectively detected and sampled with a photodetector (PD) and a digitizer. Light is managed among the EDFA2, FBG, and PD using another circulator (Cir.2).

Figure 3(a) shows 10 consecutive Rayleigh traces recorded along the SMF. At the start of the SMF, a dead-zone in the Rayleigh signal can be clearly observed which has a high-power that saturates the PD. In contrast, after the dead-zone, the recorded Rayleigh traces look normal as expected. Applying the normalized differential method [10], the PZT2's vibration event can be well-located; however, the vibration of PZT1 cannot be identified because of the dead-zone [Fig. 3(b)]. This is because when applying the normalized differential method, the subtractions of the Rayleigh traces equal roughly zero within the dead-zone, with some random noises at the dead-zone's edges, as shown in Fig. 3(c) which presents a zoom-in image 
of the differential signal around the dead-zone. In contrast, the vibration information of the PZT2's vibration event can be easily recognized with respect to the background noise, as illustrated in the zoom-in image of the differential signal around the PZT2 location [Fig. 3(d)]. For further clarifications, Figs. 3(e) and 3(f) show the power spectra of the PZT1's and PZT2's vibration events, respectively. Spectral broadband noise is measured within the dead-zone [Fig. 3(e)], while a sharp 5-kHz vibration is sensed for the PZT2 [Fig. 3(f)]. The results of Fig. 3 highlights the dead-zone challenge in OTDR-based systems.
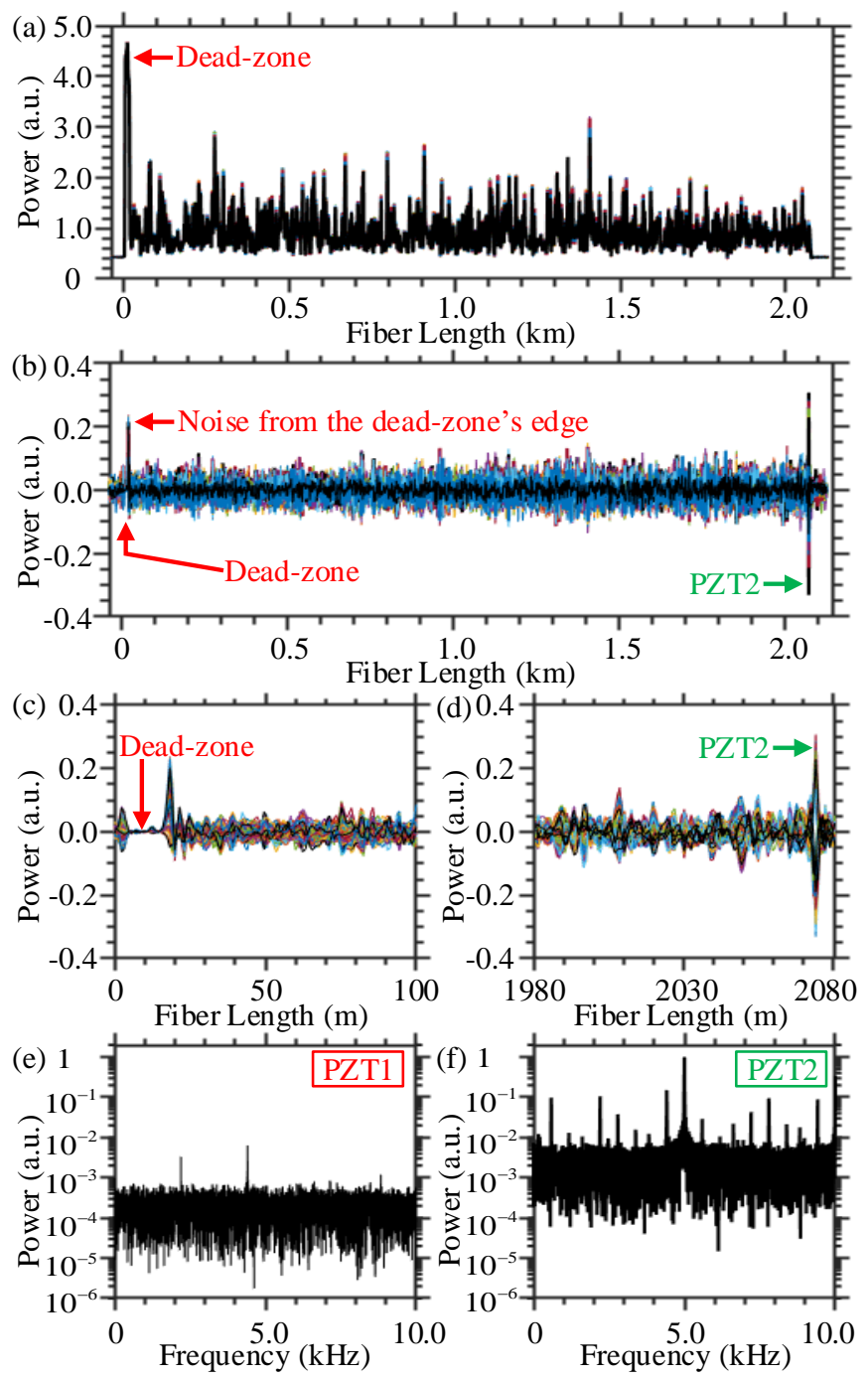

Fig. 3. 10 consecutive Rayleigh traces (a) and vibration position information (b) of the typical DAS. Zoom-in images of the vibration position information around the PZT1's (c) and PZT2's (d) location. Measured power spectra of the PZT1's (e) and PZT2's (f) vibration.

The experimental setup of the other DAS system that comprises our reported design is shown in Fig. 4, which resembles that setup in Fig. 2 with two main differences. Firstly, the FUT in Fig. 4 is a step-index TMF of a 3.27 V-number at the 1535-nm operation wavelength. Secondly, between the Cir.1 and the TMF, we add a $3 \times 1$ photonic lantern mode MUX/DEMUX of a $\sim 2.5-$ $\mathrm{dB}$ insertion loss. The insertion loss of the mode MUX/DEMUX can be compensated using the EDFA1 and/or EDFA2. The TMF here supports propagating the $\mathrm{LP}_{01}$ mode and the two degen-

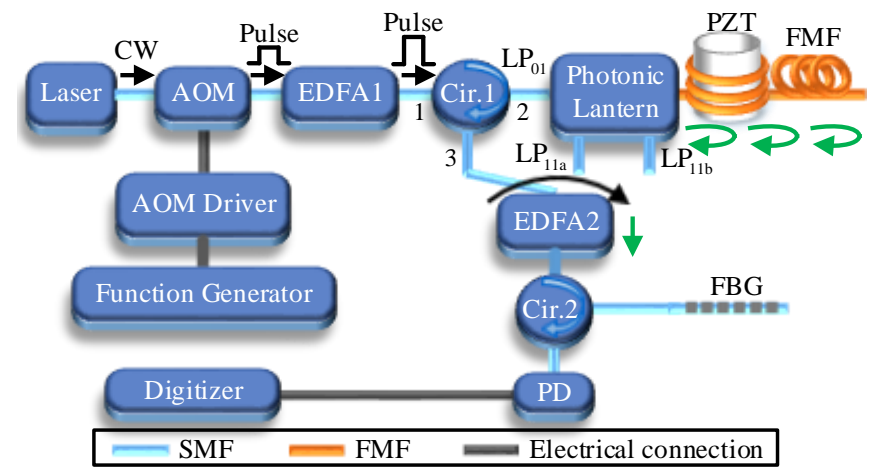

Fig. 4. Experimental setup of the OTDR-based DAS designed using a TMF and a photonic lantern.

erate $\mathrm{LP}_{11 \mathrm{a}}$ and $\mathrm{LP}_{11 \mathrm{~b}}$ modes. At a $\sim 2-\mathrm{m}$ separation from the input port of the TMF, we again wind a 2-m section of the TMF around a PZT cylinder.
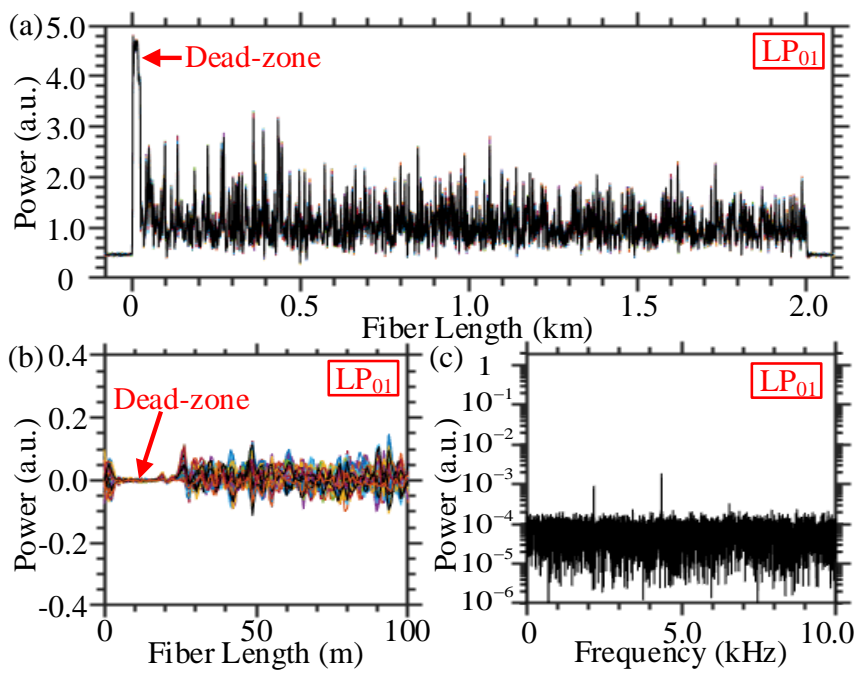

Fig. 5. 10 consecutive Rayleigh traces (a), vibration position information (b), and power spectrum of the PZT's vibration event (c) measured when collecting the Rayleigh signal associated with the $\mathrm{LP}_{01}$ mode, in the TMF-based DAS.

As a representative example, we always inject the optical pulses into the TMF through the photonic lantern's $\mathrm{LP}_{01}$ input port [Fig. 4]. We initially record the Rayleigh traces through the third port of Cir.1. These traces are associated with the backscattered Rayleigh light as $\mathrm{LP}_{01}$ mode. As expected, the $\mathrm{LP}_{01}$ mode's consecutive Rayleigh traces suffer from the dead-zone at the start of the TMF [Fig. 5(a)]. The corresponding differential signals of these Rayleigh traces, around the PZT position, are shown in Fig. 5(b) where the PZT's vibration information is missing because of the dead-zone. The power spectrum of the PZT's vibration event confirms the inability to sense the $5-\mathrm{kHz}$ vibration frequency [Fig. 5(c)]. This experimental configuration resembles that of Fig. 2; consequently, the dead-zone problem still exists.

Keeping injecting the optical pulses through the $\mathrm{LP}_{01}$ port, we next collect the Rayleigh traces from the photonic lantern's $\mathrm{LP}_{11 \mathrm{a}} / \mathrm{LP}_{11 \mathrm{~b}}$ port. Since the $\mathrm{LP}_{11 \mathrm{a}}$ and $\mathrm{LP}_{11 \mathrm{~b}}$ mode behave similarly, we present here only the results of the $\mathrm{LP}_{11 \mathrm{a}}$ mode. Fig. 6(a) shows 10 consecutive Rayleigh traces backscattered as $\mathrm{LP}_{11}$ mode. Clearly, the dead-zone disappears and the recorded 

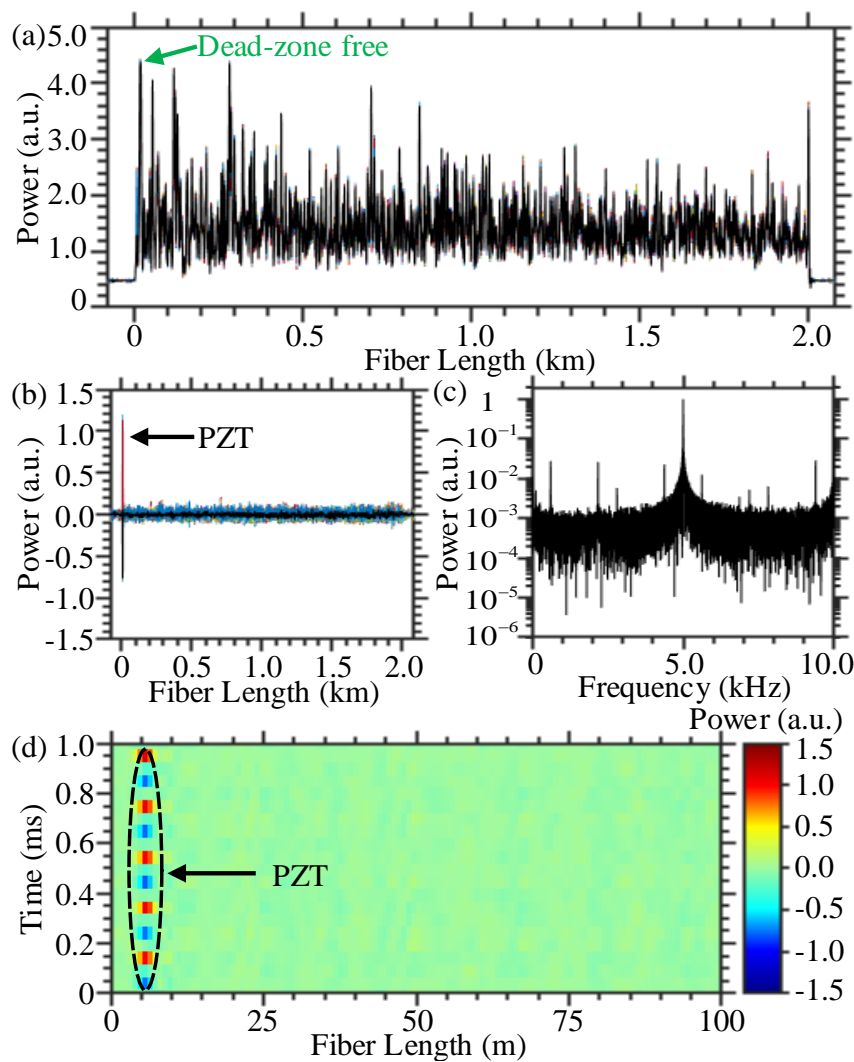

Fig. 6. 10 consecutive Rayleigh traces (a) and vibration position information (b) measured when using the $\mathrm{LP}_{11 \mathrm{a}}$ mode. (c) Power spectrum of the PZT's vibration calculated using the $\mathrm{LP}_{11 a}$ 's Rayleigh signal. (d) Temporal evolution of the normalized differential Rayleigh signal of the $\mathrm{LP}_{11 \mathrm{a}}$ mode.

Rayleigh traces do not saturate the PD along the entire TMF. Applying the normalized differential method, the vibration position information of the PZT is well-identified using the $\mathrm{LP}_{11 \mathrm{a}}$ 's Rayleigh signal, as shown in Fig. 6(b). Calculating Fourier transform of the temporal Rayleigh signal at the vibration location, Fig. 6(c) shows the power spectrum of the PZT's vibration event. The $5-\mathrm{kHz}$ vibration of the PZT is perfectly sensed when recording the Rayleigh signal from the $\mathrm{LP}_{11 \text { a }}$ port of the photonic lantern. For further clarification, Fig. 6(d) presents the temporal evolution of the normalized differential signal which obviously contrasts the PZT's vibration with the background noise. The results of Fig. 6 validate our approach of cancelling the dead-zone originated from the fiber front facet reflection, using a TMF-based OTDR. In principle, the reported approach would efficiently work using other types of FMFs [Fig. 1].

To overcome the distal end's dead-zone of OTDR system, one can simply splice a fiber optic isolator with the output port of the sensing optical fiber. In this work, we focus only on bypassing the fiber front facet's Fresnel reflection which is challenging to cancel and it generates the longest dead-zone along the FUT.

It is worth mentioning that, in the reported system, relatively expensive components such as the mode MUX/DEMUX are used. It is common in critical sensing applications that the measurand to be more important than the system's cost. The added cost in our system should be acceptable, given the advantage of accessing the sensing information within the dead-zone. As expected for most emerging technologies, the cost of the FMF and mode MUX/DEMUX would become more affordable when the market volume increases. Besides, the high insertion loss of the mode MUX/DEMUX is another challenge in our system; however, this loss can be compensated with the EDFA1/EDFA2.

In conclusion, we utilize a TMF and a mode MUX/DEMUX to overcome the OTDR's dead-zone formed by the Fresnel reflection of the fiber's front facet. The optical pulses of the OTDR are injected into the TMF using a spatial mode, while the other modes offer the sensing information along the TMF. Using this approach, a $5-\mathrm{kHz}$ vibration event created by a PZT cylinder is sensed within the dead-zone. This is achieved via using the $\mathrm{LP}_{01}$ mode for launching the pulses while collecting the Rayleigh signals of the $\mathrm{LP}_{11 \mathrm{a}} / \mathrm{LP}_{11 \mathrm{~b}}$ for the sensing purpose. Considering the expansion of using FMFs for OTDR-based sensors, the reported design would find many potential applications.

Funding Saudi Aramco (RGC/3/3138-01); King Abdullah University of Science and Technology (KAUST) (BAS/1/161401-01, GEN/1/6607-01- 01, KCR/1/2081-01-01)

Disclosures The authors declare no conflicts of interest.

\section{REFERENCES}

1. M. Tateda and T. Horiguchi, J. Light. Technol. 7, 1217 (1989).

2. X. Bao and L. Chen, Sensors (Switzerland) 12, 8601 (2012).

3. Y. Mao, I. Ashry, F. Hveding, A. Y. Bukhamsin, Y. Hong, T. K. Ng, and B. S. Ooi, IEEE J. Sel. Top. Quantum Electron. 26, 1 (2020).

4. X. Bao, D. P. Zhou, C. Baker, and L. Chen, J. Light. Technol. 35, 3256 (2017).

5. X. Ma, J. Chang, Z. Wang, W. Wang, and T. Li, IEEE Sensors J. 14, 295 (2014).

6. C. Y. Hong, Y. F. Zhang, G. W. Li, M. X. Zhang, and Z. X. Liu, "Recent progress of using Brillouin distributed fiber optic sensors for geotechnical health monitoring," (2017).

7. T. Yamate, G. Fujisawa, and T. Ikegami, J. Light. Technol. 35, 3538 (2017).

8. Filograno, Riziotis, and Kandyla, Instruments. 3, 46 (2019).

9. Z. Qin, L. Chen, and X. Bao, IEEE Photonics Technol. Lett. 24, 542 (2012).

10. I. Ashry, Y. Mao, M. S. Alias, T. K. Ng, F. Hveding, M. Arsalan, and B. S. Ooi, Appl. Opt. (2019).

11. J. C. Juarez and H. F. Taylor, Appl. Opt. 46, 1968 (2007).

12. R. J. Feuerstein, "Field measurements of deployed fiber," in Optics InfoBase Conference Papers, (2005).

13. R. Feced, M. Farhadiroushan, and V. A. Handerek, IEEE Photonics Technol. Lett. (1997).

14. M. P. Fernández, L. A. B. Rossini, J. P. Pascual, A. Pablo, and C. Caso, arXiv preprint 1801.06485, 1 (2018).

15. Y. Weng, E. Ip, Z. Pan, and T. Wang, Opt. Express 23, 9024 (2015).

16. Y. Mao, I. Ashry, M. S. Alias, T. K. Ng, F. Hveding, M. Arsalan, and B. S. Ooi, IEEE Photonics J. (2019).

17. I. Ashry, A. Wang, and Y. Xu, IEEE J. Sel. Top. Quantum Electron. (2017).

18. P. Lu, N. Lalam, M. Badar, B. Liu, B. T. Chorpening, M. P. Buric, and P. R. Ohodnicki, "Distributed optical fiber sensing: Review and perspective," (2019).

19. S. G. Leon-Saval, N. K. Fontaine, J. R. Salazar-Gil, B. Ercan, R. Ryf, and J. Bland-Hawthorn, Opt. Express (2014).

20. Z. Wang, H. Wu, X. Hu, N. Zhao, Q. Mo, and G. Li, Sci. reports 6, 1 (2016). 


\section{FULL REFERENCES}

1. M. Tateda and T. Horiguchi, "Advances in Optical Time-Domain Reflectometry," J. Light. Technol. 7, 1217-1224 (1989).

2. X. Bao and L. Chen, "Recent Progress in Distributed Fiber Optic Sensors," Sensors (Switzerland) 12, 8601-8639 (2012).

3. Y. Mao, I. Ashry, F. Hveding, A. Y. Bukhamsin, Y. Hong, T. K. Ng, and B. S. Ooi, "Simultaneous Distributed Acoustic and Temperature Sensing Using a Multimode Fiber," IEEE J. Sel. Top. Quantum Electron. 26, 1-7 (2020).

4. X. Bao, D. P. Zhou, C. Baker, and L. Chen, "Recent Development in the Distributed Fiber Optic Acoustic and Ultrasonic Detection," J. Light. Technol. 35, 3256-3267 (2017).

5. X. Ma, J. Chang, Z. Wang, W. Wang, and T. Li, "Using multiple reference points in Raman based distributed temperature sensor for eliminating DC interference," IEEE Sensors J. 14, 295-301 (2014).

6. C. Y. Hong, Y. F. Zhang, G. W. Li, M. X. Zhang, and Z. X. Liu, "Recent progress of using Brillouin distributed fiber optic sensors for geotechnical health monitoring," (2017).

7. T. Yamate, G. Fujisawa, and T. Ikegami, "Optical Sensors for the Exploration of Oil and Gas," J. Light. Technol. 35, 3538-3545 (2017).

8. Filograno, Riziotis, and Kandyla, "A Low-Cost Phase-OTDR System for Structural Health Monitoring: Design and Instrumentation," Instruments. 3, 46 (2019).

9. Z. Qin, L. Chen, and X. Bao, "Wavelet denoising method for improving detection performance of distributed vibration sensor," IEEE Photonics Technol. Lett. 24, 542-544 (2012).

10. I. Ashry, Y. Mao, M. S. Alias, T. K. Ng, F. Hveding, M. Arsalan, and B. S. Ooi, "Normalized differential method for improving the signal-to-noise ratio of a distributed acoustic sensor," Appl. Opt. (2019).

11. J. C. Juarez and H. F. Taylor, "Field test of a distributed fiber-optic intrusion sensor system for long perimeters," Appl. Opt. 46, 1968 (2007).

12. R. J. Feuerstein, "Field measurements of deployed fiber," in Optics InfoBase Conference Papers, (2005).

13. R. Feced, M. Farhadiroushan, and V. A. Handerek, "Zero dead-zone OTDR with high-spatial resolution for short haul applications," IEEE Photonics Technol. Lett. (1997).

14. M. P. Fernández, L. A. B. Rossini, J. P. Pascual, A. Pablo, and C. Caso, "DSP-Enhanced OTDR for Detection and Estimation of Events in PONs," arXiv preprint 1801.06485, 1-10 (2018).

15. Y. Weng, E. Ip, Z. Pan, and T. Wang, "Single-end simultaneous temperature and strain sensing techniques based on Brillouin optical time domain reflectometry in few-mode fibers," Opt. Express 23, 9024 (2015).

16. Y. Mao, I. Ashry, M. S. Alias, T. K. Ng, F. Hveding, M. Arsalan, and B. S. Ooi, "Investigating the Performance of a Few-Mode Fiber for Distributed Acoustic Sensing," IEEE Photonics J. (2019).

17. I. Ashry, A. Wang, and Y. Xu, "Mode-Based Reconstruction of Chemical Distributions in Optical Fibers," IEEE J. Sel. Top. Quantum Electron. (2017).

18. P. Lu, N. Lalam, M. Badar, B. Liu, B. T. Chorpening, M. P. Buric, and P. R. Ohodnicki, "Distributed optical fiber sensing: Review and perspective," (2019).

19. S. G. Leon-Saval, N. K. Fontaine, J. R. Salazar-Gil, B. Ercan, R. Ryf, and J. Bland-Hawthorn, "Mode-selective photonic lanterns for spacedivision multiplexing," Opt. Express (2014).

20. Z. Wang, H. Wu, X. Hu, N. Zhao, Q. Mo, and G. Li, "Rayleigh scattering in few-mode optical fibers," Sci. reports 6, 1-8 (2016). 